\title{
Characterization of Two-Dimensional Transition Metal Dichalcogenides in the Scanning Electron Microscope Using Energy Dispersive X-ray Spectrometry, Electron Backscatter Diffraction, and Atomic Force Microscopy
}

\author{
Christian Lang*, Matthew Hiscock, Kim Larsen, Jonathan Moffat ${ }^{1}$, Ravi Sundaram² \\ Oxford Instruments NanoAnalysis, High Wycombe HP12 3SE, UK \\ ${ }^{1}$ Asylum Research, High Wycombe HP12 3SE, UK \\ ${ }^{2}$ Oxford Instruments Plasma Technology, Yatton BS49 4AP, UK
}

*Correspondence to:

Lang $C$,

Tel: $+44(0) 1494442255$

Fax: +44 (0)1494 524129

E-mail: christian.lang@oxinst.com

Received September 17, 2015

Revised September 21, 2015

Accepted September 21, 2015
Here we show how by processing energy dispersive X-ray spectrometry (EDS) data obtained using highly sensitive, new generation EDS detectors in the AZtec LayerProbe software we can obtain data of sufficiently high quality to non-destructively measure the number of layers in two-dimensional (2D) $\mathrm{MoS}_{2}$ and $\mathrm{MoS}_{2} / \mathrm{WSe}_{2}$ and thereby enable the characterization of working devices based on $2 \mathrm{D}$ materials. We compare the thickness measurements with EDS to results from atomic force microscopy measurements. We also show how we can use electron backscatter diffraction (EBSD) to address fabrication challenges of 2D materials. Results from EBSD analysis of individual flakes of exfoliated $\mathrm{MoS}_{2}$ obtained using the Nordlys Nano detector are shown to aid a better understanding of the exfoliation process which is still widely used to produce $2 \mathrm{D}$ materials for research purposes.

Key Words: Two-dimensional materials, LayerProbe, Thickness measurement, Energy dispersive X-ray spectrometry, Electron backscatter diffraction

\section{INTRODUCTION}

Two-dimensional (2D) transition metal dichalcogenides enable exciting new applications in electronic devices and show great promise to replace traditional silicon technology as functional building blocks (Butler et al., 2013). However, in order to realize this potential there is a range of fabrication and integration challenges that have to be overcome and suitable, non-destructive characterization techniques are needed. Due to their high resolution, electron optical characterization in scanning electron microscopes (SEMs) and atomic force microscopy (AFM) is ideally suited. Also, SEM is already used extensively in the characterization and failure analysis of conventional semiconductor devices. Therefore, an extension of the same analysis technique to devices based on $2 \mathrm{D}$ materials will enable easier integration of $2 \mathrm{D}$ materials into standard production and quality control processes. Here we show how a full structural and compositional characterization can be obtained by combining energy dispersive X-ray spectrometry (EDS), electron backscatter diffraction (EBSD), and AFM analysis including the number of layers present in the $2 \mathrm{D}$ material which is critical to its performance.

\section{MATERIALS AND METHODS}

The 2D MoS${ }_{2}$ and $\mathrm{WSe}_{2}$ were produced by repeated exfoliation using adhesive tape similar to the process first

(a) This is an open-access article distributed under the terms of the Creative Commons Attribution Non-Commercial License (http://creativecommons.org/licenses/by-nc/4.0) which permits unrestricted noncommercial use, distribution, and reproduction in any medium, provided the original work is properly cited.

Copyrights @ 2015 by Korean Society of Microscopy 
described to produce graphene by Novoselov et al. (2004). The flakes were transferred onto a $300 \mathrm{~nm} \mathrm{SiO}{ }_{2}$ film which had previously been deposited onto a silicon chip. Flakes of different thickness were investigated in an optical microscope and candidates for SEM analysis identified. The SEM analysis was conducted on a Tescan Mira Field Emission SEM (Tescan, Czech) with an X-Max 150 EDS detector (Oxford Instruments, UK) and a Nordlys Nano EBSD camera (Oxford Instruments). The AFM work was carried out on an Asylum Research Cypher AFM (Oxford Instruments).

In order to measure the thickness and quantify the number of layers of the 2D material, we used the AZtec LayerProbe software (Oxford Instruments) acquire and process EDS spectra. The LayerProbe software refines a starting model of the sample structure against the EDS spectra to calculate the thickness and composition of the layers (Lang et al., 2013). The starting model comprises the layer sequence in the sample and a substrate material. In the case of the $2 \mathrm{D}$ material, the composition of the layers is known and the only unknown in the model is the layer thickness. The substrate material is $\mathrm{SiO}_{2}$.

\section{RESULTS}

The number of layers present in a 2D material is critical to its performance as with increasing number of layers the materials properties quickly converge to the bulk materials properties. As Fig. 1 and 2 show, we can obtain data of sufficiently high quality to non-destructively measure the number of layers in 2D $\mathrm{MoS}_{2}$ and $\mathrm{WSe}_{2}$ as well as from heterostructures containing both materials by processing EDS data obtained in the SEM. Fig. 1 shows an SEM image of a flake of $\mathrm{MoS}_{2}$ with two regions, one with two layers of $\mathrm{MoS}_{2}$ and one with one layer as verified by step height measurements in the AFM and by Raman spectroscopy. EDS spectra acquired from the two different regions show a clear difference in the peak height of the overlapping Mo L-lines and S K-lines. The difference can be quantified by processing the data in a special software designed to calculate the thickness of thin films on substrates (AZtec LayerProbe). For the calculation, a density of $5.06 \mathrm{~g} /$ $\mathrm{cm}^{3}$ was assumed for $\mathrm{MoS}_{2}$. The resulting values shown in Fig. 1 correspond well to a theoretical interlayer distance of 0.65 nm.

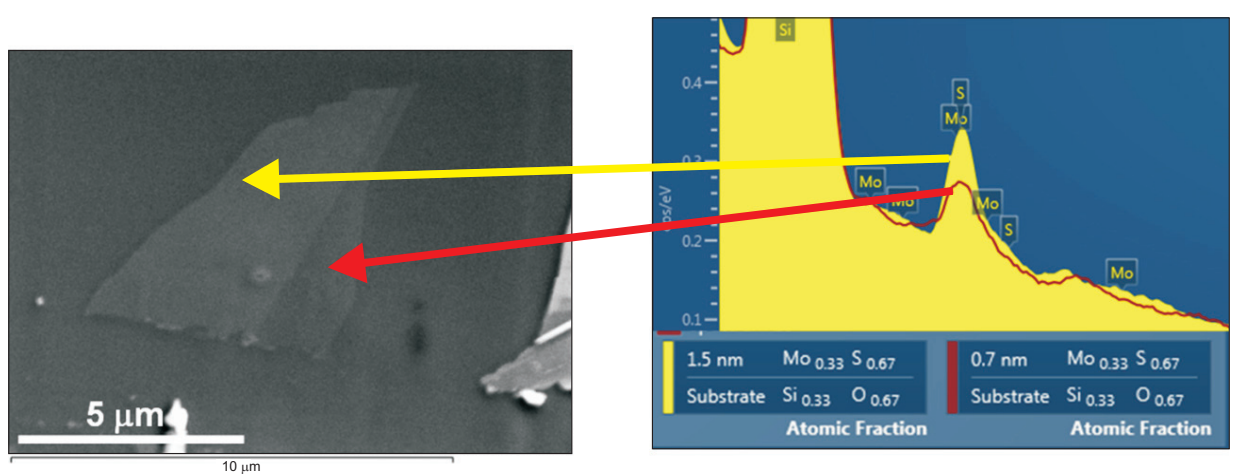

$10 \mu \mathrm{m}$
Fig. 1. A flake of $\mathrm{MoS}_{2}$ on an $\mathrm{SiO}_{2}$ substrate with EDS spectra and resulting layer thicknesses.
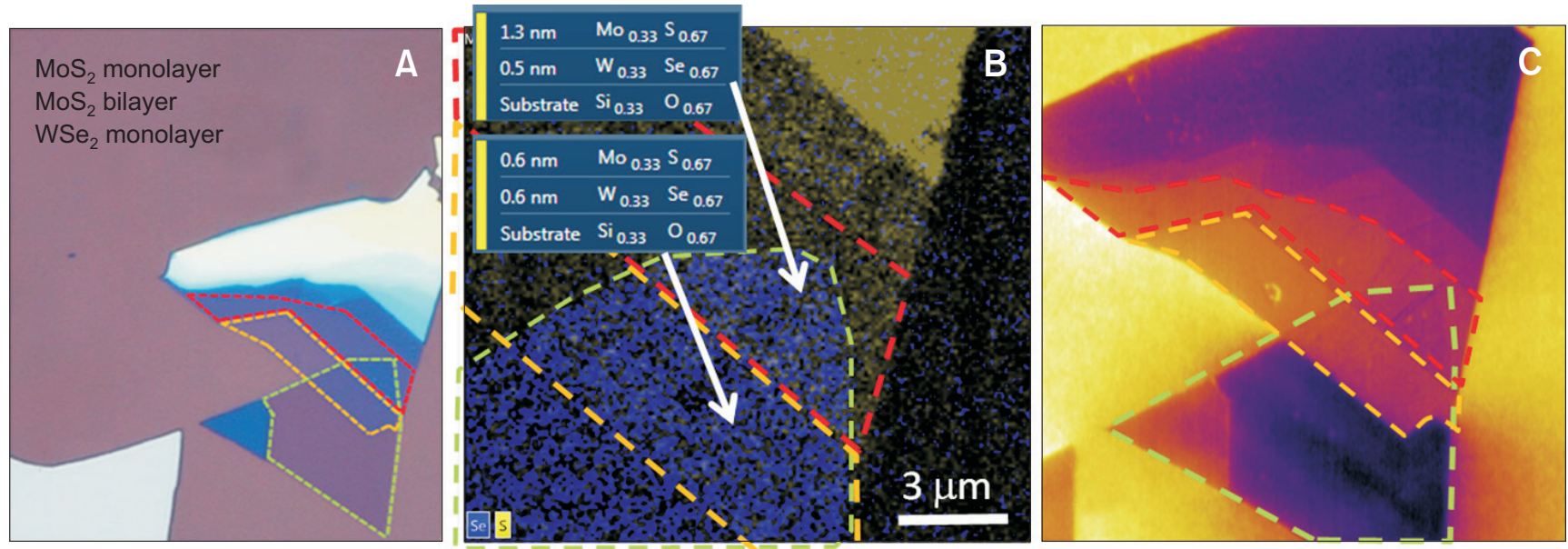

Fig. 2. (A) Optical micrograph of a $\mathrm{MoS}_{2} / \mathrm{WSe}_{2}$ heterostructure. EDS maps at $4 \mathrm{kV}$ of the overlap regions indicated by dotted lines (B) and Kelvin probe atomic force microscopy images of the overlap regions (C). 

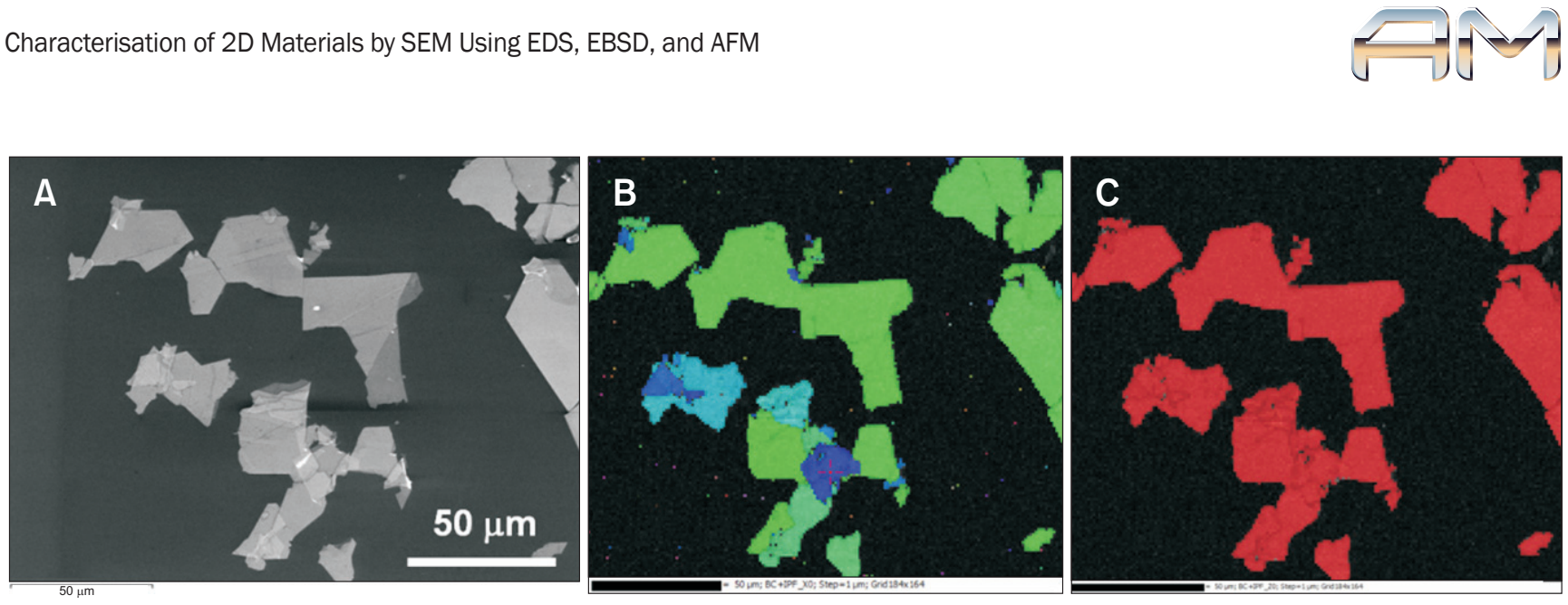

Fig. 3. Electron image (A) and inverse pole figure (IPF) x map (B) and IPF z map (C) of $\mathrm{MoS}_{2}$ flakes on $\mathrm{SiO}_{2}$ indicating only in-plane rotational misalignment of the flakes.

In order to test whether this method is also suitable for heterostructures of $2 \mathrm{D}$ materials, we obtained measurements from a sample where a flake of $\mathrm{MoS}_{2}$ had been transferred onto a flake of $\mathrm{WSe}_{2}$. In the region of where the two flakes overlap, Raman spectroscopy carried out previously on the same sample showed that while there is only a single layer of $\mathrm{WSe}_{2}$ present, $\mathrm{MoS}_{2}$ occurs in one layer and two layers. Fig. 2 indicates the different regions of interest on the sample. As the W M-line overlaps closely with the Si K-line, the Se K-line was used for the layer thickness measurement. The results in Fig. 2B show that both the $\mathrm{WSe}_{2}$ layer and the $\mathrm{MoS}_{2}$ layer thickness can be accurately determined.

We also show that Kelvin probe AFM (KPFM) can be used to image the contrast between different layer thicknesses in both single layers and heterostructures. Previous work by Choi et al. (2014) showed that KPFM can be used to probe the work function of $\mathrm{MoS}_{2}$ and that the work function increases monotonically with the number of layers. Our results in Fig. 2C show a clear differential contrast between the $\mathrm{MoS}_{2}$ and the $\mathrm{WSe}_{2}$ flakes. We also see a clear increase in the work function with the number of layers, in line with the findings of Choi et al. (2014). Interestingly, we see a reversal of that behavior for the $\mathrm{WSe}_{2}$ where the thicker part shows a significantly brighter contrast than the thinner part.

In order to add crystallographic data revealing misalignment between flakes, we can use EBSD. While differential contrast between several $\mathrm{MoS}_{2}$ flakes indicate different thicknesses (Fig. 3A), the electron image does not reveal whether different flakes are crystallographically aligned or misaligned, the former indicating that they originate from the same transfer step. An inverse pole figure (IPF) map of an area that contains several flakes of exfoliated $\mathrm{MoS}_{2}$ clearly indicates significant misalignment between some of the flakes (Fig. 3B) while also showing that the misalignment is only in the horizontal plane (Fig. 3C).

\section{DISCUSSION AND CONCLUSION}

Our results indicate the great potential of SEM and AFM for the characterization of devices based on 2D materials and indicate avenues of further work to establish them as means for failure analysis and production quality control. It establishes SEM/EDS analysis as a viable technique to measure the number of layers present in a $2 \mathrm{D}$ material and even in a heterostructure non-destructively. As both SEM/ EDS analysis is already widely available in the semiconductor industry as well as failure analysis laboratories this technique can be readily adopted when 2D materials are integrated into mass produced devices. We also showed the potential of other, complimentary analysis techniques such as KPFM and EBSD. Further work is necessary to determine whether the work function measured by KPFM can be quantified but our results show that it can be used to distinguish between $\mathrm{MoS}_{2}$ and $\mathrm{WSe}_{2}$ and confirmed that the work function of both materials depends on the number of layers present. However, we also showed that while for $\mathrm{MoS}_{2}$ the work function increases as indicated in previous reports in the literature, for $\mathrm{WSe}_{2}$ the work function is inversely proportional to the number of layers. EBSD was shown to give valuable additional information on the alignment between flakes. This may aid the understanding of the exfoliation process which is still widely used to produce 2D materials for research purposes.

\section{CONFLICT OF INTEREST}

No potential conflict of interest relevant to this article was reported. 
Lang $\mathrm{C}$ et al.

\section{REFERENCES}

Butler S Z, Hollen S M, Cao L, Cui Y, Gupta J A, Gutiérrez H R, Heinz T F, Hong S S, Huang J, Ismach A F, Johnston-Halperin E, Kuno M, Plashnitsa V V, Robinson R D, Ruoff R S, Salahuddin S, Shan J, Shi L, Spencer M G, Terrones M, Windl W, and Goldberger J (2013) Progress, challenges, and opportunities in two-dimensional materials beyond graphene. ACS Nano 7, 2898-2926.

Choi S H, Shaolin Z, and Yang W (2014) Layer-number-dependent work function of MoS2 nanoflakes. J. Korean Phys. Soc. 64, 1550-1555.
Lang C, Rühle S, Anderson A Y, Zaban A, Statham P, and Burgess S (2013) Non-destructive measurement of a combinatorial materials library for all-oxide solar cells. Microsc. Microanal. 19, 1872-1873.

Novoselov K S, Geim A K, Morozov S V, Jiang D, Zhang Y, Dubonos S V, Grigorieva I V, and Firsov A A (2004) Electric field effect in atomically thin carbon films. Science 306, 666-669. 